\title{
Le magmatisme basique hercynien et post-hercynien du Système central espagnol : essai de caractérisation des sources mantelliques
}

\author{
Carlos Villaseca ${ }^{\mathrm{a}, *}$, David Orejana ${ }^{\mathrm{a}}$, Christian Pin ${ }^{\mathrm{b}}$, José-Angel López García $^{\mathrm{c}}$, \\ Pilar Andonaegui ${ }^{a}$
}

\footnotetext{
a Departamento de Petrología y Geoquímica, Facultad Geología, UCM, 28040 Madrid, Espagne

${ }^{\mathrm{b}}$ CNRS UMR 6524, département de géologie, université Blaise-Pascal, 63038 Clermont-Ferrand, France

c Departamento de Cristalografía y Mineralogía, Facultad Geología, UCM, 28040 Madrid, Espagne
}

\section{Résumé}

Les manifestations du magmatisme basique dans le Système central espagnol s'étalent de façon sporadique sur une période de $140 \mathrm{Ma}$. Les données géochimiques suggèrent des sources mantelliques variables pour les cinq suites magmatiques reconnues. Une contribution crustale significative est mise en évidence dans les magmas Gb1 à Gb3, mis en place en liaison avec l'orogénèse hercynienne. Elle témoigne de processus d'hybridation intracrustal et/ou du recyclage de matériaux d'origine continentale dans le manteau. En revanche, le magmatisme post-collisionnel ultérieur montre une empreinte crustale négligeable dans la suite lamprophyrique alcaline Gb4, extraite d'un réservoir source de type OIB. La suite tholéiitique Gb5 pourrait témoigner d'un manteau source métasomatisé par un composant d'origine crustale.

\begin{abstract}
Hercynian and post-Hercynian basic rocks from the Spanish Central System: estimates of Central Spain subcontinental mantle. Basic magmatism in central Spain occurred sporadically during a long period of ca. 140 Ma. The geochemical data on representative samples from the five igneous episodes show that different mantle reservoirs were tapped. Crustal involvement (via recycling in mantle sources or by hybridization at emplacement levels) was important in the basic magmas related to the Hercynian orogeny (Gb1 to Gb3 suites). Samples occurring as later dykes have signatures ranging from OIB-like values (Gb4 alkaline lamprophyres) to those of a formerly enriched subcontinental lithospheric mantle (tholeiites Gb5).
\end{abstract}

Mots-clés : roches basiques ; manteau sous-continental; géochimie ; isotopes du $\mathrm{Sr}$ et $\mathrm{Nd}$; Système central espagnol

Keywords: basic rocks; subcontinental mantle; geochemistry; (Sr, Nd) isotopes; Spanish Central System 


\section{Abridged English version}

\section{Introduction}

Several episodes of basic magmatism occurred sporadically during a long period of about $140 \mathrm{Ma}$ in the Spanish Central System (SCS). Five groups of basic rocks can be distinguished (Table 1). A first group (Gbl), emplaced from 345 to $310 \mathrm{Ma}[4,5,8]$, corresponds to gabbros to quartzdiorites in small massifs, coeval with the huge SCS granitic Hercynian batholith (Fig. 1). They show a medium-K calc-alkaline character $[4,16,27]$. The next four suites are exposed as late-stage dyke-swarms crosscutting the SCS batholith (Fig. 1). The Gb2 and Gb3 dykes are associated with granite porphyries forming a complex acid-basic association [13]. They range from medium-high-K calcalkaline compositions (Gb2 types) to shoshonitic types (Gb3 types) emplaced during post-collisional stages, from 295 to $245 \mathrm{Ma}$ [12]. An extensional setting is proposed for these subvolcanic groups [13]. Laterstage alkaline lamprophyres and associated alkaline monzogabbro-to-syenite porphyries form the Gb4 igneous suite of rather uncertain age, around 280 $235 \mathrm{Ma}[4,19,26]$. A time-integrated depleted mantle source, enriched in LREE and $\mathrm{Nb}-\mathrm{Ta}$ during postHercynian rifting is suggested for that group [4]. Finally, the tholeiitic Messejana-Plasencia great dyke was emplaced ca. $200 \mathrm{Ma}$ ago in the western part of the SCS $[11,22]$, in relation with the opening of the central Atlantic. This last Gb5 magmatism reflects a mantle source enriched with recycled crustal components [1] and complex assimilation processes during segregation through the crust [9]

\section{Whole rock geochemistry}

Most of studied rocks are strictly basic (or ultrabasic, for some lamprophyres), with the exception of Gb2 dykes (Fig. 2). Moreover, all these mantlederived samples have lost their primary character mainly by crystal fractionation (as most rocks occur as phenocryst-rich varieties: Gb2-3-4 types). For that reason, criteria relatively insensitive to fractional crystallization such as incompatible trace element ratios and radiogenic $\mathrm{Sr}-\mathrm{Nd}$ data are used to draw inferences on their mantle sources [15,29]. Nevertheless, some hybrid petrographic features in $\mathrm{Gbl}-\mathrm{Gb} 2-\mathrm{Gb} 3$ rocks make a precise assessment of their original source chemistry more difficult to achieve.

Alkalinity increases progressively from $\mathrm{Gbl}$ to Gb4 types; Gb1 and Gb2 rocks plot in the subalkaline (calc-alkaline) field, whereas Gb3 and Gb4 samples plot in the alkaline field (Fig. 2) with potassic character: shoshonitic and shoshonitic/ultrapotassic, respectively. Samples from the last magmatic event (Gb5) show a low-K subalkaline (tholeiitic) character (Fig. 2).

Primitive-mantle-normalized incompatible trace element data (Fig. 3) display two different patterns: a first group (Gb1, Gb2, Gb3 and Gb5) is characterized by negative $\mathrm{Nb}$, Ta and Ti anomalies, and positive $\mathrm{Pb}$ anomalies, which are similar to those of subductionrelated rock suites, and the continental crust, in general; a second group (Gb4) displays smoother pattern, closer to those of OIB-like melts, with apparently negligible crustal contribution. Some trace element ratios sensitive to the involvement of continental crust material, either as recycled components in the mantle sources, or during ascent through the crust $(\mathrm{Ce} / \mathrm{Pb}$, $\mathrm{Nb} / \mathrm{U}, \mathrm{Th} / \mathrm{Yb}, \mathrm{Ta} / \mathrm{Yb}$ ) (Fig. 4), also reflect a strong crustal imprint in Gb1, Gb2 and Gb3 magmas. This signature is much weaker in the tholeiitic Gb5 gabbros, and negligible in the alkaline Gb4 lamprophyres.

New Sr-Nd isotopic data (Table 2) show that Gbl, Gb2 and Gb3 groups plot in the more enriched part of an age-corrected ${ }^{87} \mathrm{Sr} /{ }^{86} \mathrm{Sr}$ vs. ENd diagram, from BSE-like values towards the field for typical continental crustal rocks (Fig. 5). Alkaline lamprophyres (Gb4) overlap the isotope field of OIP [29], from values of strongly depleted mantle on a time-integrated basis (with high $\varepsilon_{\mathrm{Nd}}$ and low ${ }^{87} \mathrm{Sr} /{ }^{86} \mathrm{Sr}$ initial ratios: +6.2

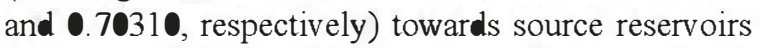
that were slightly enriched on a time-integrated basis ( -1.0 and 0.7045 , respectively). Finally, the tholeiitic gabbros Gb5 plot in enriched fields with a fairly

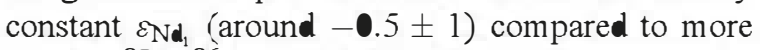
variable ${ }^{87} \mathrm{Sr} /{ }^{86} \mathrm{Sr}_{\mathrm{i}}(\mathbf{0} .7052-\mathbf{0 . 7 0 7 7})[1,9]$, as often observed in continental tholeiites.

\section{Discussion}

The geochemical and radiogenic isotope data set available on the SCS basic magmatism show that the subcontinental mantle beneath central Spain is markedly heterogeneous. Radiogenic isotope data fluc- 
tuate between involvement (via recycling in mantle sources or by hybridisation at emplacement levels, or both) was important in the basic magmas related to the Hercynian orogeny. Introduction of crustal material in $\mathrm{Gbl}-\mathrm{Gb} 2$ $\mathrm{Gb} 3$ suites is well exemplified by their low $\mathrm{Ce} / \mathrm{Pb}$ and $\mathrm{Nb} / \mathrm{U}$, as these incompatible element ratios are very sensitive to the incorporation of materials from the continental crust. This is in agreement with previous studies on some of these basic suites $[4,16,18]$. More primitive compositions of Gb3 gabbros preclude major contributions of crustal material during magma ascent and favour source enrichment.

Lithospheric rifting was involved during the last two basic igneous episodes $[1,4,7,9,26]$. Alkaline lamprophyres $\mathrm{Gb} 4$ also define an heterogeneous compositional field similar in trace element ratios and $\mathrm{Sr}-\mathrm{Nd}$ isotope signatures to IB-type magmas. The tholeiitic gabbros Gb5 define an enriched compositional field similar to that of the Gb1-Gb2-Gb3 suites, but their incompatible trace element ratios are less 'crustal', whereas higher initial $\varepsilon_{\mathrm{Nd}}$ values suggest, either a lower conibution from components recycled from the continental crust, or the involvement of crustal materials with more radiogenic $\mathrm{Nd}$ ratios (lower crust) [1,9]. Their close compositional similarity with the large igneous province of low-Ti tholeiitic basalts of the Cenral Atlantic Magmatic Province (CAMP) [20] is noticeable.

The compositional range of subcrustal mantle in central Spain is similar to that defined for Western Europe during Cenozoic times [6], and is suggestive of a long stable period of the central parts of the Iberian plate after the major lithosphere-forming Hercynian cycle

\section{Introduction}

L'étude des magmas basiques permet, dans une certaine mesure, d'entrouvrir une «fenêtre » sur le manteau sous-jacent. Dans le Système central espagnol (SCE), plusieurs épisodes de mise en place de magmas basiques d'origine mantellique se sont succédé depuis l'orogénèse hercynienne (Carbonifère) (Fig. 1).

Le premier groupe de roches basiques (Gbl) est constitué par un ensemble de petits massifs de gabbros à diorites quartziques, mis en place de manière précoce ou en même temps que les granitoïdes peralumineux qui composent le batholite du SCE, et avec lesquels ils ont interagi de diverses manières $[4,16,27]$. Les âges radiométriques de $312 \pm 8$ Ma pour le massif de El Arenal ( $\mathrm{Pb} / \mathrm{Pb}$ sur mono-cristal de zircon) [5], de $322 \pm 5$ Ma pour le massif de El Tiemblo (isochrone $\mathrm{Rb}-\mathrm{Sr}$ ) [8], ou de de $34 \pm 18$ Ma pour les gabbros de Gredos (isochrone $\mathrm{Rb}-\mathrm{Sr}$ ) [4], sont en accord avec le caractère précoce de ces massifs vis-à-vis du reste du batholite du SCE. Ce magmatisme est de nature calcoalcaline, avec des concentrations moyennes en $\mathrm{K}$, et ses caractéristiques isotopiques $\mathrm{Sr}-\mathrm{Nd}[4,16]$ attestent la présence d'un composant crustal significatif.

Les deux suites de magmas basiques ultérieures sont post-batholitiques et appartiennent à des champs filoniens de porphyres, associés spatialement aux plutons granitiques [13]. On y distingue une première suite clairement calco-alcaline avec des teneurs élevées en $\mathrm{K}_{2} \bullet(\mathrm{Gb} 2)$, et une suite plus récente d'affinité shoshonitique ou monzonitique (Gb3). Les rares datations radiométriques correspondent à des âges approximatifs de $290 \pm 10 \mathrm{Ma}$ (isochrone $\mathrm{Rb}-\mathrm{Sr}$ des porphyres granitiques associés) et $245 \pm 7 \mathrm{Ma}(\mathrm{K}-$ $\mathrm{Ar}$ en roche totale, a priori moins fiable), respectivement [12].

Le réseau de filons alcalins postérieur (Gb4) montre une plus grande complexité chimique. Sur la base des données géochronologiques assez imprécises actuellement disponibles, certains de ces filons semblent s'être mis en place dès le Permien inférieur (isochrone $\mathrm{Rb}-$ Sr à $283 \pm 30$ Ma obtenue par Bea et al. [4], en bon accord avec l'âge $\mathrm{K}-\mathrm{Ar}$ à $277 \pm 7 \mathrm{Ma}$ d'une phlogopite séparée d'un lamprophyre, F. Stuart, pers. comm.). Toutefois, l'absence de magmatisme granitique associé, le niveau de mise en place très superficiel, sous forme de diatrèmes, de certains filons [26], ainsi que l'âge triasique assigné, au Portugal, à d'autres filons basiques alcalins du secteur Centro-Ibérique (Portugal Ferreira et Regencio Macedo [19] présentent des âges $\mathrm{K}-\mathrm{Ar}$ dans l'intervalle 235-207 Ma), pourraient suggérer une chronologie de mise en place plus complexe, couvrant une longue période. Plusieurs sousséries alcalines peuvent être distinguées au sein de cet ensemble Gb4 : lamprophyres ultrabasiques, lamprophyres phlogopitiques, diabases et porphyres gabbroïques (Tableau 1) $[4,26]$. Une contribution crustale négligeable et une source de type manteau enrichi mé- 


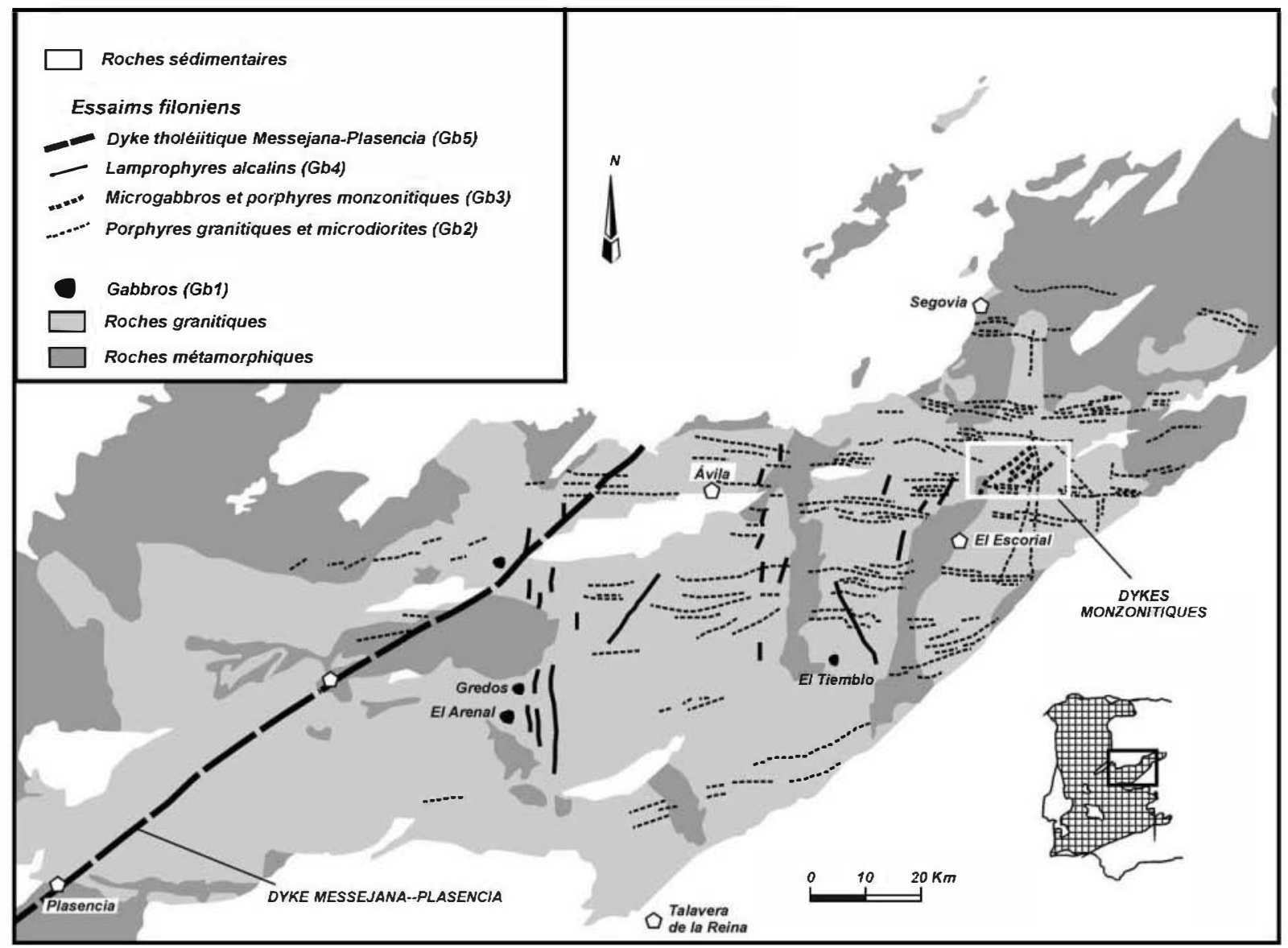

Fig. 1. Cadre géologique simplifié du Système central espagnol montrant les massifs de gabbros Gb1 et les champs filoniens de roches basiques ultérieurs : Gb2 (calco-alcaline), Gb3 (monzonitique), Gb4 (alcaline) et Gb5 (tholéiitique).

Fig. 1. Geological sketch of the Spanish Central System showing Gb1 gabbroic massifs and subsequent mafic dyke swarms: Gb2 (calc-alkaline), Gb3 (shoshonite), Gb4 (alkaline) and Gb5 (tholeiitic).

tasomatiquement ont été proposées pour ce magmatisme lamprophyrique [4].

La manifestation la plus tardive du magmatisme basique dans ce secteur est le grand dyke de MessejanaPlasencia, qui raverse la péninsule Ibérique depuis sa marge atlantique sud-occidentale. Daté d' autour de $200 \mathrm{Ma}[11,22]$, ce grand corps filonien se rattache à la province centro-atlantique mésozoïque. Il est composé de gabbros tholéiitiques à quartz normatif, contenant un composant crustal, dont l'origine, par recyclage au niveau de la source mantellique ou bien par contamination lors de la mise en place filonienne, a fait l'objet de discussions $[1,7,9]$.

Ce ravail a pour but de présenter une synthèse des données géochimiques et isotopiques ( $\mathrm{Sr}, \mathrm{Nd}$ ), pu- bliées ou obtenues par notre groupe, relatives à ces épisodes magmatiques basiques successifs, qui témoignent de la composition du manteau sous-continental du centre de l'Espagne pendant quelque $140 \mathrm{Ma}$.

\section{Sélection des données}

La base de données analytiques relative aux roches ignées basiques dans le Système central n'est pas très fournie. Nous avons choisi les échantillons pour lesquels une documentation géochimique la plus complète possible était disponible, non seulement pour les éléments majeurs, mais aussi pour les éléments en traces (LILE, HFSE, REE) et les données isotopiques. 
Nous avons completé ces données géochimiques et réalisé 11 nouvelles analyses isotopiques $\mathrm{Sr}-\mathrm{Nd}$ (Tableau 2). En général, on a sélectionné les termes les plus primitifs des séries, qui dans tous les cas ont été interprétés comme d'origine mantellique, en écartant les analyses correspondant à des roches cumulatives ainsi que les échantillons manifestement d' origine hybride, ou ayant subi des processus d'altération. Toutefois, les roches basiques des séries Gbl-2-3 montrent un léger et variable degré de contamination crustale (par exemple, xénocristaux de quartz et/ou plagioclases avec zonation spongieuse), qui rend plus délicate la discussion sur les sources mantelliques de ces roches. Aucun des échantillons sélectionnés ne présente les caractéristiques de magma primaire; dans tous les cas, les teneurs en $\mathrm{Cr}(<540 \mathrm{ppm}), \mathrm{Ni}$ $(<176 \mathrm{ppm})$ et l' indice $\mathrm{mg}(\mathrm{Mg} /(\mathrm{Mg}+\mathrm{Fe}))(<\bullet, 70)$ sont relativem entfaibles (Tableau 2). De fait, quelques types (Gb2), avec des concentrations en silice de 53$55 \%$, ne sont pas des roches basiques, bien que leur teneur en $\mathrm{Mg} \bullet$ dépasse généralement $5 \%$. La majorité des échantillons a subi une cristallisation fractionnée évidente antérieurement à la mise en place, en accord avec le caractère plus ou moins porphyrique de la plupart des séries filoniennes (Gb2, 3 et 4). Cependant, la séparation de minéraux mafiques (olivine, pyroxènes), bien qu' elle explique que les compositions primaires de ces magmas n'aient pas été préservées, ne modifierait pas substantiellement les rapports d'éléments en traces incompatibles. Pour cette raison, la plus grande partie de la discussion suivante sera principalement basée sur les rapports $\mathbf{d}$ 'isotopes radiogéniques et d'éléments en traces rès incompatibles, les plus susceptibles de refléter ceux des sources mantelliques.

\section{Caractérisation du magmatisme basique}

Les échantillons sélectionnés ont été reportés dans le diagramme $\mathrm{Si}_{2}-\left(\mathrm{Na}_{2} \boldsymbol{-}+\mathrm{K}_{2} \boldsymbol{\bullet}\right)$. On observe (Fig. 2) le caractère basique de la majorité de roches, hormis les filons microdiorítiques (Gb2) de nature intermédiaire, et quelques filons alcalins (Gb4), de composition ultrabasique. Le degré d'alcalinité croît depuis les termes calco-alcalins précoces $(\mathrm{Gbl})$ et microdioritiques ( $\mathrm{Gb} 2$ ), vers les gabbros modérément alcalins (monzonitiques, $\mathrm{K}_{2} \bullet>\mathrm{Na}_{2} \bullet$, [13]), mais à né- 
Tableau 2

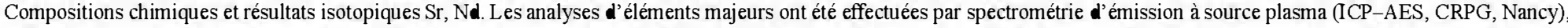

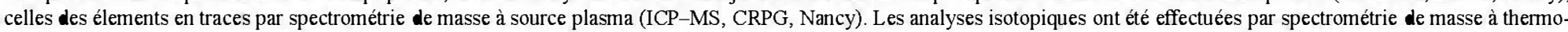

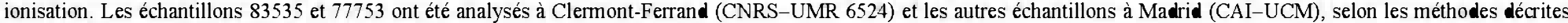
dans les références [17] et [28]

Table 2

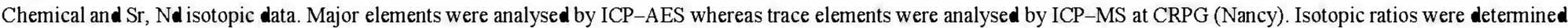
by TIMS at CNRS-UMR 6524 (Clermont-Ferrand) (samples 83535 and 77753) and at CAI-UCM (Madrid). Isotopic analytical methods after [17] and [28]

\begin{tabular}{|c|c|c|c|c|c|c|c|c|c|c|c|}
\hline & Gb1 & Gb2 & & Gb3 & & Gb4 & & & & Gb5 & \\
\hline Échantillon & 83535 & 82556 & 82558 & 82566 & 81277 & 77753 & 81938 & 103473 & 103674 & 103309 & 103325 \\
\hline $\mathrm{SiO}_{2}$ & 52,13 & 53,88 & 53,85 & 48,5 & 49,29 & 41,2 & 46,96 & 45,66 & 43,62 & 51,47 & 51,59 \\
\hline $\mathrm{TiO}_{2}$ & $\bullet, 61$ & 0,96 & $\bullet, 89$ & 1,61 & 1,45 & 2,75 & 1,86 & 3,61 & 3,36 & 1,06 & 1,00 \\
\hline $\mathrm{Al}_{2} \mathrm{O}_{3}$ & 18,22 & 16,69 & $17, \mathbf{0 0}$ & 16,64 & 16,42 & 13,28 & 16,81 & 16,68 & 15,21 & 15,28 & 14,70 \\
\hline $\mathrm{Fe}_{2} \mathrm{O}_{3}$ & 7,06 & 7,99 & 8,20 & 9,26 & 8,95 & 12,33 & 11,83 & 12,39 & 11,82 & 9,96 & 11,02 \\
\hline $\mathrm{MnO}$ & $\mathbf{0}, 12$ & $\bullet, 14$ & $\bullet, 14$ & $\bullet, 15$ & $\mathbf{0}, 15$ & $\bullet, 16$ & $\bullet, 15$ & $\bullet, 12$ & $\bullet, 15$ & $\bullet, 17$ & $\bullet, 17$ \\
\hline $\mathrm{MgO}$ & 8,29 & 6,37 & 5,86 & 5,33 & 6,59 & 11,05 & 4,50 & 4,91 & 7,00 & 6,37 & 7,25 \\
\hline $\mathrm{CaO}$ & 8,56 & 6,33 & 7,61 & 6,69 & 7,03 & 10,24 & 5,95 & 5,07 & 8,57 & 10,74 & 11,10 \\
\hline $\mathrm{Na}_{2} \mathrm{O}$ & 2,15 & 2,58 & 2,94 & 3,72 & 3,08 & 1,18 & 3,13 & 2,97 & 2,66 & 2,28 & 2,07 \\
\hline $\mathrm{K}_{2} \mathrm{O}$ & $\bullet, 98$ & 1,89 & 1,35 & 2,63 & 3,11 & 3,52 & 2,58 & 4,39 & 3,10 & $\bullet, 67$ & $\bullet, 67$ \\
\hline $\mathrm{P}_{2} \mathrm{O}_{5}$ & $\bullet, 12$ & $\bullet, 16$ & $\bullet, 13$ & $\bullet, 59$ & $\mathbf{0}, 47$ & $\bullet, 58$ & $\bullet, 61$ & $\bullet, 78$ & $\bullet, 53$ & $\bullet, 11$ & $\bullet, 11$ \\
\hline P.F. & 1,61 & 3,04 & 1,48 & 4,51 & 3,38 & 3,31 & 5,28 & 3,58 & 4,00 & $\bullet, 66$ & $\bullet, 52$ \\
\hline Total & 99,85 & 100,01 & 99,43 & 99,63 & 99,91 & 99,60 & 99,66 & 100,16 & 100,02 & 98,76 & 100,2 \\
\hline $\mathrm{Ba}$ & 241 & 505 & 371 & 1190 & 114 & 854 & 576 & 2475 & 963 & 147 & 138 \\
\hline $\mathrm{Rb}$ & 39 & 105 & 58 & 139 & 144 & 74 & 50 & 137 & 91 & 25 & 28 \\
\hline $\mathrm{Sr}$ & 172 & 235 & 173 & 663 & 532 & 429 & 653 & 803 & 88 & 186 & 179 \\
\hline $\mathrm{Zr}$ & 85 & 141 & 133 & 229 & 201 & 167 & 214 & 367 & 263 & 91 & 85 \\
\hline $\mathrm{Nb}$ & 11 & 7 & 5,7 & 26,3 & 22,1 & 69 & 59 & 110 & 81,5 & 6,2 & 5,4 \\
\hline $\mathrm{Ta}$ & & $\bullet, 49$ & $\bullet, 36$ & 1,99 & 1,67 & & & 8,87 & 6,34 & $\bullet, 36$ & $\bullet, 33$ \\
\hline Th & 2 & 5,41 & 2,97 & 17,4 & 14,1 & 5 & 4 & 6,21 & 5,26 & 1,68 & 1,48 \\
\hline $\mathrm{U}$ & 2 & $\bullet, 81$ & $\bullet, 54$ & 3,15 & 2,32 & & & 1,75 & 1,19 & $\mathbf{0}, 42$ & $\bullet, 35$ \\
\hline Hf & & 3,8 & 3,5 & 5,3 & 4,8 & & & 7,61 & 5,85 & 2,4 & 2,30 \\
\hline $\mathrm{Pb}$ & 10 & 27 & 6 & 29 & 30 & & & 6,4 & 5,3 & 4 & 3,6 \\
\hline $\mathrm{Y}$ & 17 & 24,2 & 25,1 & 33,2 & 28,8 & 28,5 & 33,1 & 66,2 & 24,6 & 20,7 & 20,1 \\
\hline $\mathrm{La}$ & 12,12 & 20,20 & 14,30 & 71,00 & 64,30 & 39,67 & 40,37 & 82,30 & 44,00 & 9,62 & 8,57 \\
\hline $\mathrm{Ce}$ & 28,97 & 41,00 & 29,7 & 135,00 & 123,00 & 69,71 & 71,57 & 137,00 & 92,10 & 20,8 & 18,5 \\
\hline $\mathrm{Nd}$ & 12,80 & 21,90 & 16,30 & 60,40 & 57,50 & 35,20 & 31,68 & 84,40 & 42,90 & 12,5 & 11,20 \\
\hline $\mathrm{Sm}$ & 2,86 & 4,84 & 3,85 & $10,3 \bullet$ & 10,00 & 7,12 & 6,91 & 16,90 & 7,45 & 3,14 & 2,95 \\
\hline $\mathrm{Eu}$ & 1,05 & 1,25 & 1,15 & 2,68 & 2,40 & 2,27 & 2,19 & 4,64 & 2,43 & 1,14 & 1,04 \\
\hline $\mathrm{Gd}$ & 2,50 & 4,92 & 4,22 & 8,69 & 8,02 & 6,56 & 6,06 & 14,6 & 6,54 & 3,75 & 3,48 \\
\hline Dy & 2,35 & 4,74 & 4,66 & 6,58 & 5,87 & 4,90 & 5,27 & 11,9 & 4,76 & 4,07 & 3,85 \\
\hline $\mathrm{Er}$ & 1,29 & 2,63 & 2,74 & $3,5 \bullet$ & 3,03 & 2,39 & 3,37 & 5,85 & 2,33 & 2,34 & 2,27 \\
\hline
\end{tabular}



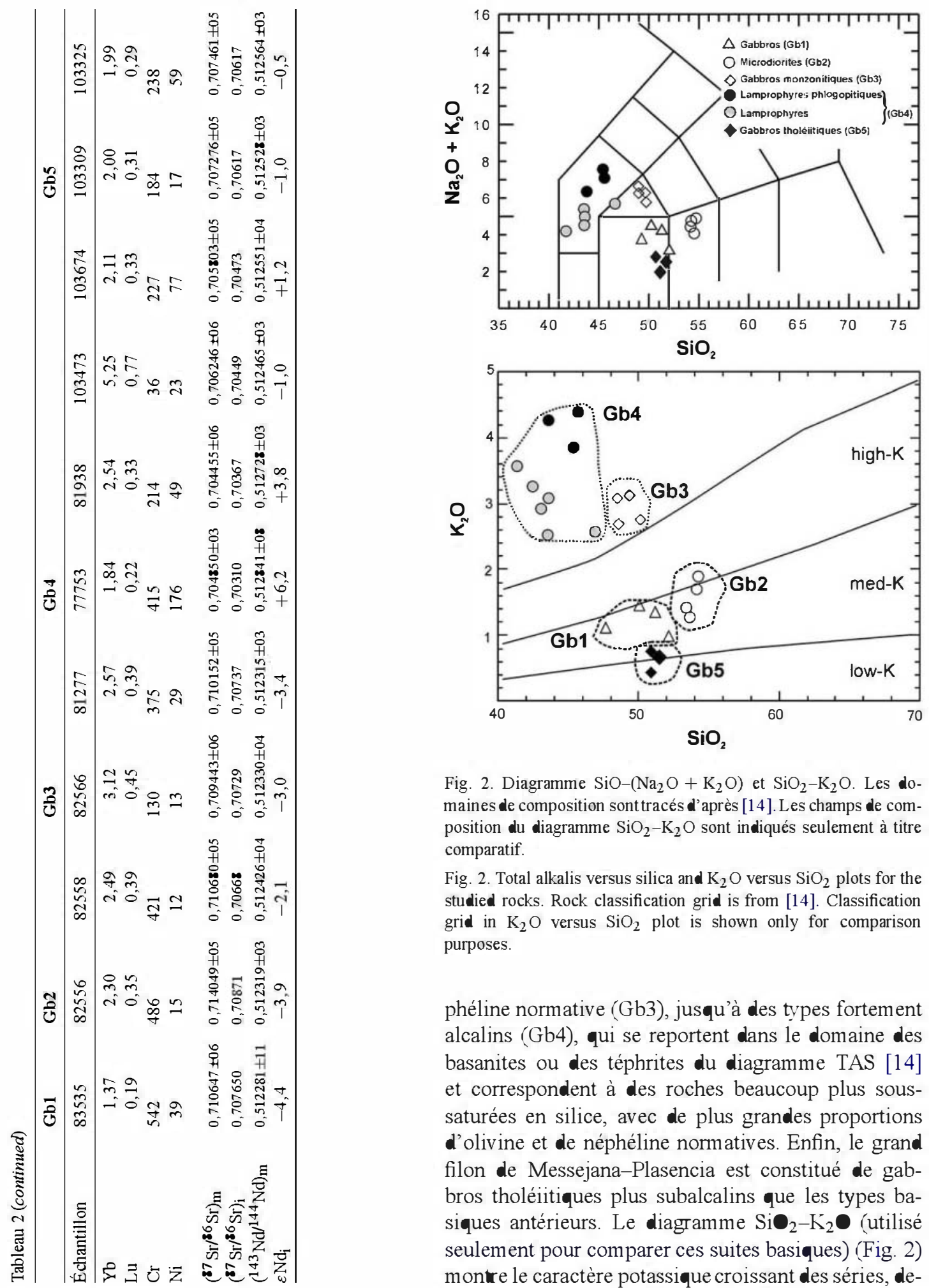

Fig. 2. Diagramme $\mathrm{SiO}-\left(\mathrm{Na}_{2} \mathrm{O}+\mathrm{K}_{2} \mathrm{O}\right)$ et $\mathrm{SiO}_{2}-\mathrm{K}_{2} \mathrm{O}$. Les domaines de composition sont tracés d'après [14]. Les champs de composition du diagramme $\mathrm{SiO}_{2}-\mathrm{K}_{2} \mathrm{O}$ sont indiqués seulement à titre comparatif.

Fig. 2. Total alkalis versus silica and $\mathrm{K}_{2} \mathrm{O}$ versus $\mathrm{SiO}_{2}$ plots for the studied rocks. Rock classification grid is from [14]. Classification grid in $\mathrm{K}_{2} \mathrm{O}$ versus $\mathrm{SiO}_{2}$ plot is shown only for comparison purposes.

phéline normative (Gb3), jusqu'à des types fortement alcalins (Gb4), qui se reportent dans le domaine des basanites ou des téphrites du diagramme TAS [14] et correspondent à des roches beaucoup plus soussaturées en silice, avec de plus grandes proportions d'olivine et de néphéline normatives. Enfin, le grand filon de Messejana-Plasencia est constitué de gabbros tholéiitiques plus subalcalins que les types basiques antérieurs. Le diagramme $\mathrm{Si}_{2}-\mathrm{K}_{2}$ (utilisé seulement pour comparer ces suites basiques) (Fig. 2) montre le caractère potassique croissant des séries, de- 


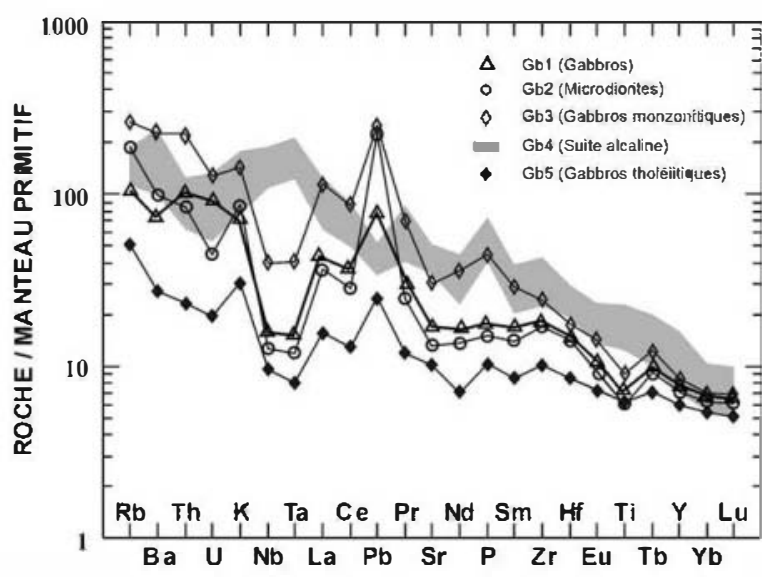

Fig. 3. Profils, normalisés aux valeurs du manteau primitif, des éléments en traces incompatibles pour une sélection de roches basiques. Les profils correspondent aux valeurs moyennes d'échantillons représentatifs des gabbros $\mathrm{Gbl}(n=4)$, des roches microdioritiques $\mathrm{Gb} 2(n=4)$, des gabbros monzonitiques Gb3 $(n=4)$, des lamprophyres alcalins Gb4 (domaine grisé, $n=6$ ) et des diabases tholéiitiques $\mathrm{Gb5}(n=3)$. Les valeurs de normalisation sont celles de [24], sauf pour $\mathrm{P}$ et $\mathrm{Pb}$, empruntées à la référence [23].

Fig. 3. Distribution of incompatible trace elements, normalized to primordial mantle values [24], except $\mathrm{P}$ and $\mathrm{Pb}$ values after [23]. The patterns are for averaged values of $\mathrm{Gb} 1$ gabbros $(n=4), \mathrm{Gb} 2$ microdiorites $(n=4), \mathrm{Gb} 3$ monzonitic gabbros $(n=4)$, and Gb5 tholeiitic diabases $(n=3)$. The shaded field is for $\mathrm{Gb} 4$ alkaline lamprophyres $(n=6)$

puis les gabbros tholéiitiques $\mathrm{Gb5}$, en passant par les types calco-alcalins (Gbl-2) et monzonitiques (Gb3), jusqu'aux lamprophyres (Gb4), qui sont reportés dans les domaines plus potassiques.

\section{Géochimie des éléments en traces}

Les profils normalisés de distribution des éléments en traces (Fig. 3) montrentune certaine similitude pour tous les types basiques à l'exception de ceux de la série alcaline (Gb4), nettement différents. Des anomalies négatives en $\mathrm{Nb}-\mathrm{Ta}$ (et $\mathrm{Ti}$ pour $\mathrm{Gbl}, \mathrm{Gb} 2$ et $\mathrm{Gb} 3$ ) et positives en $\mathrm{Pb}$, typiques des suites magmatiques en relation avec des processus de subduction ou affectées par des interactions avec la croûte continentale, sont présentes dans tous les cas, hormis les filons alcalins Gb4.

Dans la plupart des diagrammes, les matériaux alcalins (Gb4) sont situés dans le champ des magmas exraits d'une source mantellique enrichie en éléments incompatibles de type E-MORB et OIB. Les rapports $\mathrm{Th} \mathrm{Yb}$ plus élevés des gabbros monzonitiques et calco-alcalins ( $\mathrm{Gb} 3, \mathrm{~Gb} 2$ et $\mathrm{Gb}$ ) suggèrent une contributioncrustale plus importante dans ces magmas que dans les autres types (Fig. 4). Ceci est confirmé pour les faibles valeurs des rapports $\mathrm{Ce} / \mathrm{Pb}, \mathrm{Nb} / \mathrm{U}$ (Fig. 4) et $\mathrm{P} / \mathrm{Nd}$, et les valeurs élevées de $\mathrm{La} / \mathrm{Nb}$ et $\mathrm{Th} \mathrm{Nb}$. Sur cette base, la participation d'un composant crustal peut être considérée comme négligeable dans la suite alcaline $\mathrm{Gb} 4$, mais significative pour les tholéiites Gb5. L'augmentation progressive du rapport $\mathrm{La} / \mathrm{Nb}$ depuis de faibles valeurs, typiques des magmas de type intraplaque non contaminés, dans les filons alcalins Gb4 (1) et les tholéiites Gb5 (1,2), jusqu'à des valeurs supérieures à 3 dans les gabbros monzonitiques $\mathrm{Gb3}$, indique aussi une participation importante de composants crustaux dans ces magmas basiques Gbl-2-3.

\section{Géochimie isotopique}

Les données isotopiques $\mathrm{Sr}-\mathrm{Nd}$ montrent aussi une forte variabilité (Fig. 5). Au moins trois composants distincts du point de vue isotopique sont nécessaires pour expliquer la diversité des magmas basiques étudiés. Un premier composant aurait une signature $\mathrm{Sr}-\mathrm{Nd}$ voisine de celle d'un réservoir modèle hypothétique, aux caractéristiques isotopiques primitives (Bulk Silicate Earth, BSE). Il correspond à certains gabbros calco-alcalins hercyniens (Gbl) affleurant tant dans le SCE $[4,8,16]$ que dans la région voisine de Tolède [3]. La majorité des magmas basiques hercyniens de la zone centro-ibérique ont des compositions isotopiques proches des valeurs de ce réservoir modèle BSE ou légèrement plus enrichies (par exemple, [10]). Les gabbros Gbl, plus radiogéniques en $\mathrm{Sr}$, ont été affectés par des processus d'hybridation avec des magmas granitiques crustaux (par exemple, [16]). Les données isotopiques $\mathrm{Sr}-\mathrm{Nd}$ des matériaux basiques calco-alcalins $\mathrm{Gb} 2$ et $\mathrm{Gb} 3$ se reportent dans le même quadrant représentatif des réservoirs enrichis du diagramme $\varepsilon_{\mathrm{Sr}}-\varepsilon_{\mathrm{Nd}}$, mais leurs rapports ${ }^{87} \mathrm{Sr} /{ }^{86} \mathrm{Sr}$ sont sensiblement plus radiogéniques (de 0,7067 à 0,7087) que ceux des gabbros Gb1. Ces suites Gb2 et Gb3 occupent un domaine très semblable à celui défini par d'autres roches basiques calco-alcalines tardi- 

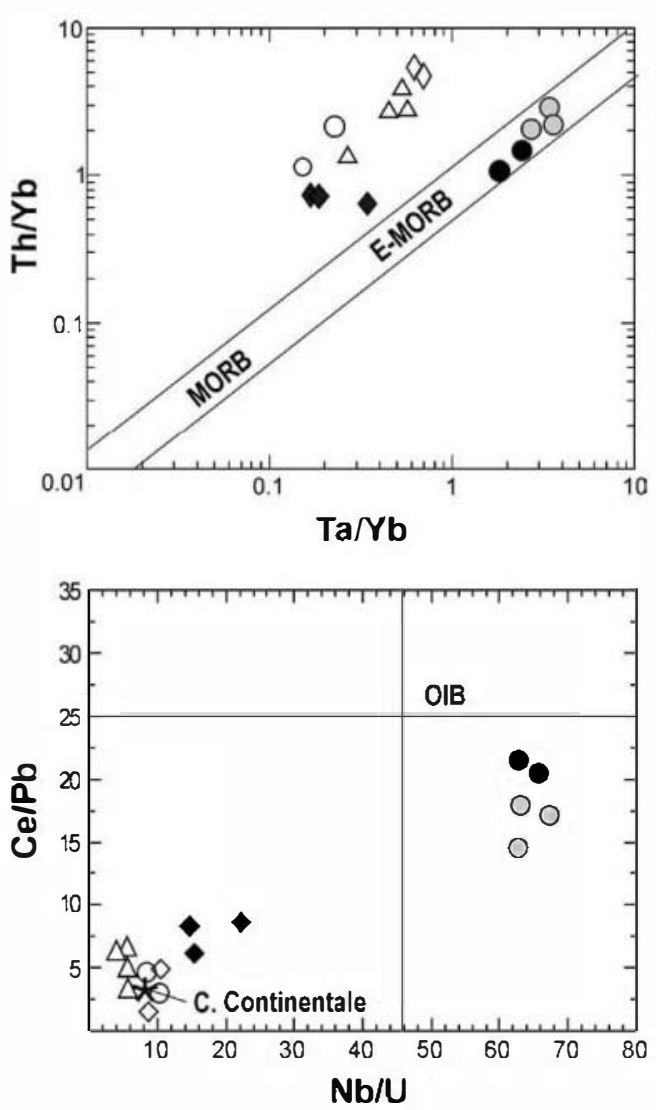

Fig. 4. Diagrammes impliquant des rapports d'éléments en traces à comportement incompatible lors des processus de fusion partielle de roches mantelliques. Même légende que pour la Fig. 1. Les domaines représentatifs des N-MORB, E-MORB, OIB et de la croûte continentale moyenne sont figurés d'après les références [15, 29].

Fig. 4. Variation of incompatible element abundance ratios for the studied samples. Same legend as for Fig. 1. Compositional fields for N-MORB, E-MORB, OIB and 'continental crust' are taken from $[15,29]$.

hercyniennes [2,21] ou par les lamprophyres d'Europe occidentale [25].

Le second composant est défini par les lamprophyres (camptonites) ultrabasiques alcalins (Gb4), qui se situent le long du mantle array, dans la partie appauvrie du champ des IP (Fig. 5). Ces données isotopiques contrastent nettement avec l'enrichissement marqué en Terres Rares légères de ces lamprophyres, qui requiert, même si l'on admet qu'ils sont issus d'un faible degré de fusion partielle, une source de type enrichi au moment de la production des magmas.

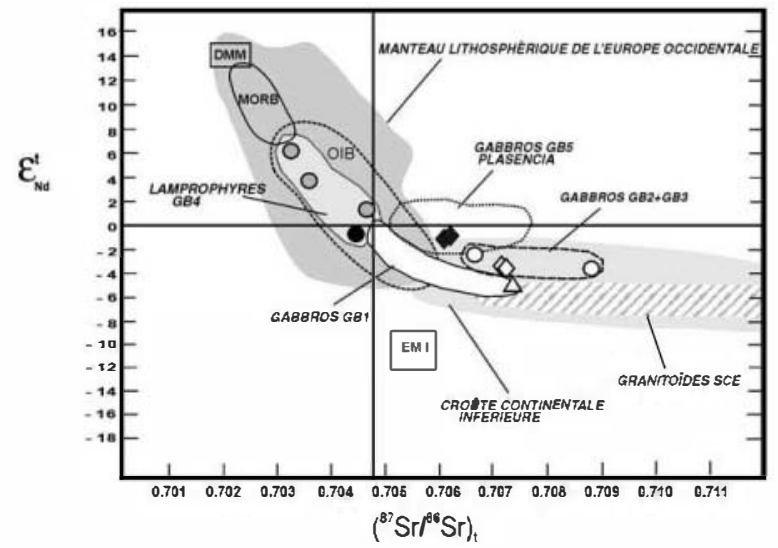

Fig. 5. Diagramme $\varepsilon_{\mathrm{N}}$ vs. $\left({ }^{87} \mathrm{Sr} /{ }^{86} \mathrm{Sr}\right)_{\mathrm{i}}$ pour les échantillons étudiés (Tableau 2). Le domaine des gabbros calco-alcalins Gb1 du SCE est figuré avec les données isotopiques des références [3, $4,8,16]$. Les données pour les diabases tholéiitiques Gb5 [1,9] sont aussi figurées. Le champ du «manteau lithosphérique de l'Europe occidentale» est dessiné d'après [6]. Les données relatives aux granitoïdes du SCE et aux échantillons de la croûte continentale inférieure de ce secteur sont extraites des références $[27,28]$ et calculées pour un âge de 300 Ma. Les réservoirs isotopiques DMM, MORB, OIB et EM-I sont d'après [6,29].

Fig. 5. $\varepsilon_{\mathrm{N}}$ vs. $\left({ }^{87} \mathrm{Sr} /{ }^{86} \mathrm{Sr}\right)_{\mathrm{i}}$ diagram for basic rocks (see Table 2). The field for Gbl calkalkaline gabbros includes data from $[3,4,8$, 16]. Data for Gb5 tholeiites (Plasencia dyke) from $[1,9]$ are plotted. The field for the 'Western Europe lithospheric mantle' is from [6]. Fields for granitoids and lower crustal rocks from the SCE, calculated at $300 \mathrm{Ma}$, are from [27,28]. Fields for DMM, MORB, OIB and EM-I are from $[6,29]$.

est donc conduit à envisager un processus d'enrichissement de la source mantellique par un composant à fortes teneurs en éléments inès incompatibles, s'étant produit peu de temps avant ou lors de l'épisode magmatique à l'origine de la suite Gb4. D'aurres données isotopiques sur les filons de camptonites [4], correspondant à des valeurs rès proches de la BSE, suggèrent que le composantresponsable de l'enrichissement de la source était lui-même hétérogène du point de vue isotopique.

Enfin, les gabbros tholéiitiques Gb5 du dyke de Plasencia montrent des valeurs relativement constantes des rapports initiaux du néodyme ( $\left.\varepsilon_{\mathrm{N} d} \approx \mathbf{0}\right)$, associées à une dispersion beaucoup plus grande des rapports

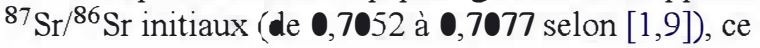
qui suggère un découplage des deux systèmes isotopiques, caractérisé par un ajout sélectif de Sr radiogénique, soit aux magmas tholéiitiques au cours de leur 
raversée de la croûte continentale, soit à leur source mantellique.

\section{Discussion}

L'étude géochimique des séries basiques mises en place dans le SCE sur une période de plus de $140 \mathrm{Ma}$, depuis les stades collisionnels hercyniens jusqu'à l'époque du riffing lié à l'ouverture de l'Atlantique au Mésozoïque, permet de mettre en évidence l'hétérogénéité de composition du manteau sous-continental de cette région. Dans l'ensemble, les données isotopiques démontrent le caractère enrichi de ce manteau souscontinental par des composants crustaux recyclés, en relation avec des événements de subduction anciens. Un certain degré de contamination par la croûte continentale pendant la traversée et la mise en place de ces magmas doit être envisagé pour les gabbros Gb1, Gb2 et $\mathrm{Gb} 3$. En revanche, un manteau appauvri de longue date, mais ayant subi un enrichissement très récent, probablement par les produits d'une fusion partielle limitée du manteau sous-jacent, est indiqué pour les lamprophyres alcalins $\mathrm{Gb} 4$, pour lesquels on peut exclure une participation significative de tout matériel crustal. L'introduction d'un composant crustal est évidente dans les gabbros $\mathrm{Gbl}, \mathrm{Gb} 2$ et $\mathrm{Gb} 3$, qui montrent de faibles valeurs des rapports $\mathrm{Ce} / \mathrm{Pb}$ et $\mathrm{Nb} / \mathrm{U}$ (Fig. 4), rès sensibles à l'incorporation de matériel crustal, et de moindre importance pour les gabbros Gb5. Le caractère plus basique et primitif des gabbros $\mathrm{Gb} 3 \mathrm{com}$ parés aux gabbros $\mathrm{Gb} 1$ et Gb2, ainsi que leurs teneurs plus élevées en REE et LILE, rendent plus difficile l'interprétation des signatures isotopiques en termes d'hybridation crustale pendant la mise en place. Par conséquent, l'existence de sources enrichies dans le manteau semble envisageable.

Les gabbros $\mathrm{Gbl}$ montrent des preuves géologiques claires d'hybridation avec les magmas granitiques contemporains, ce qui rend compte de l'étalement des données vers le domaine de composition typique des granites (Fig. 5). Au sein de ce groupe, les gabbros de Tolède (La Bastida, [3]) sont les faciès les moins affectés par ces processus hybridation tardive. Leur signature isotopique suggère une dérivation à partir d'un réservoir mantellique de type $\mathrm{BSE}$, déjà largement invoqué dans les modèles de mélange élaborés pour expliquer la pétrogénèse de ces massifs gabbroïques et les enclaves microgrenues mafiques des granitoïdes du SCE $[4,8,16,18]$, sans toutefois préjuger de la signification géologique précise de cette signature, l'existence de domaines primitifs dans le manteau supérieur étant improbable.

Les gabbros Gb5 ne montrent pas d'indice pétrographique d'hybridation avec des matériaux crustaux au niveau de leur mise en place. Pour cette raison, l'influence de phénomènes de recyclage crustal dans la source mantellique a été suggérée [1]. Plus récemment, l'hypothèse a été avancée d'une interaction directe entre un magma extrait d'un réservoir du manteau préalablement enrichi et des matériels de la croûte inférieure [9]. Les données isotopiques du $\mathrm{Pb}$ disponibles pour ces gabbros tholéiitiques $\left({ }^{206} \mathrm{~Pb} /{ }^{204} \mathrm{~Pb}=\right.$ $18,4-18,7$ et $\left.{ }^{207} \mathrm{~Pb} /{ }^{204} \mathrm{~Pb}=15,6[1,9]\right)$, qui tendent vers des sources de type EMII, paraissent plus en accord avec l'in roduction de matériaux crustaux recyclés dans la source mantellique [1]. Les basaltes tholéiitiques pauvres en $\mathrm{Ti}$ de la grande province magmatique centre-atlantique ont la même composition en éléments en traces et la même signature isotopique que le dyke de Messejana-Plasencia $[1,9,2 \mathbf{0}]$.

Le troisième pôle isotopique, représenté par les lamprophyres alcalins, paraît peu ou pas affecté par l'ajout de composants crustaux, comme le suggère l'absence d'anomalies négatives en $\mathrm{Nb}-\mathrm{Ta}$ ou positives en $\mathrm{Pb}$ dans les diagrammes normalisés des éléments incompatibles de ces roches (Fig. 3). Cette suite alcaline présente, tant du point de vue isotopique $\mathrm{Sr}-$ Nd que du point de vue des éléments incompatibles, de nombreuses similitudes avec les basaltes intraplaques de type OIB : rapports $\mathrm{Ce} / \mathrm{Pb}, \mathrm{Nb} / \mathrm{U}, \mathrm{P} / \mathrm{Nd}, \mathrm{Nb} / \mathrm{Y}$, $\mathrm{Th} / \mathrm{Yb}, \mathrm{Ce} / \mathrm{Yb}$ élevés associés à des rapports La/Nb, $\mathrm{Ba} / \mathrm{Nb}, \mathrm{Y} / \mathrm{Nb}$ et $\mathrm{Zr} / \mathrm{Nb}$ faibles (Fig. 4) $[15,29]$. Pourtant, la suite Gb4 est elle-même hétérogène, comme en témoignent non seulement la variabilité des compositions isotopiques, mais aussi les différences de rapports d'éléments en traces incompatibles.

Par ailleurs, on discerne une certaine évolution au cours du temps de la composition chimique du manteau du SCE échantillonné par les différentes suites magmatiques basiques. Le magmatisme basique associé à l'orogénèse hercynienne (types Gbl à Gb3), y compris lors des stades tardifs, montre une forte empreinte crustale, reflétant, soit des processus de contamination intra-crustale, soit la fusion de domaines mantelliques contenant une composante crustale recy- 
clée, les deux explications n'étant pas exclusives l'une de l'aure. Le magmatisme basique post-hercynien, mis en place en contexte distensif et étroitement lié à la fracturation de la lithosphère centro-ibérique, témoigne de l'implication de réservoirs mantelliques moins hybridés. Les magmas Gb4, de type lamprophyrique, semblent extraits de sources mantelliques appauvries de longue date et enrichies, peu de temps avant leur extraction, par un composant lui-même mantellique de type alcalin, riche en Terres Rares légères et en $\mathrm{Nb}-\mathrm{Ta}$. En revanche, les magmas tholéiitiques Gb5 montrent une signature (enrichissement en Sr radiogénique, anomalies négatives en $\mathrm{Nb}-\mathrm{Ta}$ ) atribuable à un manteau métasomatisé par un composant riche en fluides aqueux et/ou liquides silicatés, probablement lors d'épisodes de subduction anciens (antérieurs à la collision hercynienne, voire protérozoïque), suivi d'une faible assimilation ( $<10 \%$ vol.) de granulites de la croûte inférieure [9].

Enfin, on peut noter que la gamme de composition isotopique ( $\mathrm{Sr}-\mathrm{Nd})$ du manteau sous-continental du centre de l'Espagne, du Carbonifère au Jurassique, coïncide, dans les grandes lignes, avec celle observée en Europe occidentale au Cénozoïque [6] (Fig. 5), ce qui reflète vraisemblablement une histoire comparable et la relative stabilité de ces régions après l'événement lithosphérique majeur hercynien.

\section{Remerciements}

Nous sommes reconnaissants au Dr. H. Berrand et à un autre rapporteur anonyme pour leurs lectures critiques du manuscrit et leurs suggestions constructives. Nous remercions le Dr. M.J. Huertas de nous avoir facilité l'étude des échantillons des roches Gb2 et Gb3. Ce ravail a été subventionné par le ministère espagnol de la Science et de la Technologie (projet BTE20000575).

\section{Références}

[1] C. Alibert, A Sr-Nd isotope and REE study of Late Triassic dolerites from the Pyrenees (France) and the Messejana dyke (Spain and Portugal), Earth Planet. Sci. Lett. 73 (1985) 81-90.

[2] P. Atzori, R. Cirrincione, A. Del Moro, P. Mazzoleni, Petrogenesis of Late Hercynian calc-alkaline dykes of mid-eastem Sar- dinia: petrographical and geochemical data constraining hybridization process, Eur. J. Mineral. 12 (2000) 1261-1282.

[3] L. Barbero, G. Rogers, Geochemical and isotopic (Sr, Nd) constraints on the origin of the calc-alkaline syn-orogenic association from the Anatectic Complex of Toledo (Hercynian Iberian Belt), Geogaceta 20 (1996) 703-706.

[4] F. Bea, P. Montero, J.F. Molina, Mafic precursors, peraluminous granitoids, and late lamprophyres in the Avila Batolith a model for the generation of Variscan batholiths in Iberia, J. Geol. 107 (1999) 399-419.

[5] F. Bea, P. Montero, J.H. Scarrow, J.F. Molina, The Gredos sector of the Avila Batholith, Central Iberia: an introduction (Gredos Seminar on crustal granites), Universidad de Granada, Spain, 2002, $30 \mathrm{p}$.

[6] L. Beccaluva, G. Bianchini, M. Coltorti, W.T. Perkins, F. Siena, C. Vaccaro, M. Wilson, Multistage evolution of the European lithospheric mantle: new evidence from Sardinian peridotite xenoliths, Contrib. Mineral. Petrol. 142 (2001) 284-297.

[7] H. Bertrand, G. Millot, Le magmatisme tholéitique continental de la marge Ibérique, précurseur de l'overture de l'Atlantique central : les dolérites du dyke de Messejana-Plasencia (Portugal-Espagne), C. R. Acad. Sci. Paris, Ser. II 304 (1987) 215-220.

[8] R. Casillas, Y. Vialette, M. Peinado, J.-L. Duthou, C. Pin, Âges et caractéristiques isotopiques ( $\mathrm{Sr}, \mathrm{Nd}$ ) des granitoïles de la Sierra de Guadarrama occidentale (Espagne), Abstract Séance spécialisée Soc. géol. France mém. Jean Lameyre, 1991

[9] J.M. Cebriá, J. López-Ruiz, M. Doblas, L.T. Martins, J. Munha, Geochemistry of the Early Jurassic Messejana-Plasencia dyke (Portugal-Spain); implications on the origin of the Central Atlantic Magmatic Province, J. Petrol. 44 (2003) $547-$ 568.

[10] G. Dias, J. Leterrier, The genesis of felsic-mafic plutonic associations: a Sr and Nd isotopic study of the Hercynian Braga Granitoids Massif (Northern Portugal), Lithos 32 (1994) 207223.

[11] A.M. Dunn, P.H. Reynolds, D.B. Clarke, J.M. Ugidos, A comparison of the age and composition of the Shelburne dyke, Nova Scotia, and the Messejana dyke, Spain, Can. J. Earth Sci. 35 (1998) 1110-1115.

[12] C. Galindo, M.J. Huertas, C. Casquet, Cronología Rb-Sr y $\mathrm{K}-\mathrm{Ar}$ de diques de la Sierra de Guadarrama (Sistema Central Español), Geogaceta 16 (1994) 23-26.

[13] M.J. Huertas, C. Villaseca, Les derniers cycles magmatiques posthercyniens du Système central espagnol : les essaims filoniens calco-alcalins, Schweiz. Mineral. Petrogr. Mitt. 74 (1994) 383-401

[14] R.W. Le Maitre, P. Bateman, A. Dudek, J. Keller, J. Lameyre, M.J. Le Bas, P.A. Sabine, R. Schmid, H. Sorensen, A. Streckeisen, A.R. Wooley, B. Zanettin, Igneous rocks: a classification and glossary of terms, Cambridge University Press, Cambridge, 2002, $236 \mathrm{p}$

[15] R. McDonald, N.W. Rogers, J.G. Fitton, S. Black, M. Smith, Plume-lithosphere interactions in the generation of the basalts of the Kenya rift, East Africa, J. Petrol. 42 (2001) 877-900.

[16] I. Moreno-Ventas, G. Rogers, A. Castro, The role of hybridization in the genesis of Hercynian granitoids in the Gredos Mas- 
sif, Spain: inferences from Sr-Nd isotopes, Contrib. Mineral. Petrol. 120 (1995) 137-149.

[17] C. Pin, J.F. Santos Zalduegui, Sequential separation of light rare-earth elements, thorium and uranium by miniaturized extraction chromatography: application to isotopic analyses of silicate rocks, Anal. Chim. Acta 339 (1997) 79-89.

[18] L. Pinarelli, A. Rottura, Sr and Nd isotopic study and Rb-Sr geochronology of the Bejar granites, Iberian Massif, Spain, Eur. J. Mineral. 7 (1995) 577-589.

[19] M. Portugal Ferreira, C.A. Regêncio Macedo, Actividade magmática durante o Mesozoico: I-achega para a dataçao $\mathrm{K}$ Ar das rochas filonianas básicas intrusivas da zona CentroIbérica (Portugal), Memor. Notic. Publ. Lab. Mineral. Geol. Univ. Coimbra 87 (1979) 29-49.

[20] C. Rapaille, A. Marzoli, H. Bertrand, G. Féraud, L. Reisberg, D. Fontignie, Geochemistry and age of the European CAMP basalts, Geochim. Cosmochim. Acta 66 (2002) A625 (abstr.).

[21] R.L. Romer, H.J. Förster, C. Breitkreuz, Intracontinental extensional magmatism with a subduction fingerprint: the Late Carboniferous Halle Volcanic Complex (Germany), Contrib. Mineral. Petrol. 141 (2001) 201-221

[22] A. Sebai, G. Féraud, H. Bertrand, J. Hanes, ${ }^{40} \mathrm{Ar} /{ }^{39} \mathrm{Ar}$ dating and geochemistry of tholeiitic magmatism related to the early opening of the Central Atlantic rift, Earth Planet. Sci. Lett. 104 (1991) 455-472.

[23] S.S. Sun, Lead isotopic study of young volcanic rocks from mid-ocean ridges, ocean islands and island arcs, Phil. Trans. R. Soc. Lond. A 297 (1980) 409-445.

[24] S.R. Taylor, S.M. McLennan, The continental crust: its composition and evolution, Blackwell, Oxford, UK, 1985, $312 \mathrm{p}$.

[25] L. Turpin, D. Velde, G. Pinte, Geochemical comparison between minettes and kersantites from the Westem European Hercynian orogen: trace element and $\mathrm{Pb}-\mathrm{Sr}-\mathrm{Nd}$ isotope constraints on their origin, Earth Planet. Sci. Lett. 87 (1988) 73-86.

[26] C. Villaseca, J. Nuez, Diques camptoníticos en el Sistema Central Español, Estud. Geol. 42 (1986) 69-77.

[27] C. Villaseca, L. Barbero, G. Rogers, Crustal origin of Hercynian peraluminous granitic batholiths of Central Spain: petrological, geochemical and isotopic $(\mathrm{Sr}, \mathrm{Nd})$ constraints, $\mathrm{Li}$ thos 43 (1998) 55-79.

[28] C. Villaseca, H. Downes, C. Pin, L. Barbero, Nature and composition of the lower continental crust in central Spain and the granulite-granite linkage: inferences from granulitic xenoliths, J. Petrol. 40 (1999) 1465-1496.

[29] M. Wilson, Igneous petrogenesis, Unwin Hyman, London, $1989,466 \mathrm{p}$ 